\title{
Endometrial Hyperplasia: Emergence of The EIN System
}

\author{
Rajalakshmi.V*, Rajeswari .k, MeenakshiSundaram and Sathish Selvakumar.A \\ Department of Pathology, ESIC Medical College \& PGIMSR, Chennai, Tamilnadu, India
}

\section{ABSTRACT}

Background: The diagnosis of precancerous lesions of the endometrium remains unstandardized because, the existing World Health Organization classification categories do not correspond to distinctive biologic groups and are inadequately supported by reproducible histopathologic criteria. The objective of our study is to simplify the diagnosis of endometrial hyperplasia, make it more reproducible and stratify patients in to two risk groups instead of four using the recent EIN system.

Materials \& Methods: All cases diagnosed as endometrial hyperplasia classified according to the WHO system in patients with abnormal uterine bleeding, during the 3 year period 2014-2016 were reclassified as benign hyperplasia and Endometrial intraepithelial neoplasia according to the EIN system.

Results: 38 out of the 46 cases (82.6\%) of simple hyperplasia were reclassified as Benign hyperplasia. 8 out of the 46 cases (17.4\%) of simple hyperplasia were reclassified as EIN.1 case of complex hyperplasia without atypia was reclassified as EIN. All the 11 cases of complex hyperplasia with atypia were reclassified as EIN.

Conclusions: Application of the criteria for EIN successfully segregates patients into high and low cancer risk subgroups with better reproducibility than $\mathrm{WHO}$ classification.

Keywords: Endometrial intra epithelial neoplasia, Endometrial hyperplasia, EIN.

\section{Introduction}

Endometrial intraepithelial neoplasia (also known as 'EIN') is a precursor to endometrioid endometrial adenocarcinoma characterized by monoclonal growth of mutated cells, a distinctive histopathologic appearance, and 45-fold elevated cancer risk. ${ }^{[1,2,3]} \mathrm{EIN}$ arises through complex interactions involving the sequential accumulation of genetic damage in endometrial glands and the positive selective pressure of unopposed estrogen. EIN is to be distinguished from adenocarcinoma and the diffuse hormonal changes of EH seen in anovulation. ${ }^{[4,5]}$

Endometrial hyperplasia is a common disease with incidence of $15 \%$ in patients with abnormal intrauterine bleeding. Because only $1-28 \%$ of hyperplasias actually progress to malignant disease, depending on the degree of severity, it is important to stratify patients into high-risk and low-risk groups before initiating therapy. The World Health Organization (WHO) 1994 classification system for endometrial hyperplasias (WHO94) is used widely for this purpose. The most recent WHO classification system, the EIN system, acknowledges the shortcomings of WHO94 and, on this basis, has introduced the alternative molecular genetics-based and morphometric-based Endometrial Intraepithelial Neoplasia (EIN) classification system. ${ }^{[6]}$
The two classification systems differ in their foundations. The WHO94, which is based entirely on histologic findings, uses four subcategories based on architectural and cytologic alterations. In practice, the diagnostic criteria are difficult to apply reproducibly, because they largely are subjective. Even acknowledged experts experience substantial differences in reporting. Data from the Gynecologic Oncology Group showed that the WHO94 diagnostic criteria were misinterpreted easily in the community, because $30 \%$ of specimens that were submitted as complex atypical hyperplasia $(\mathrm{CAH})$ were diagnostic of malignancy on expert review, whereas another $40 \%$ of specimens were a lesser degree of hyperplasia or were entirely benign. ${ }^{[7]}$

The EIN system, in contrast, has a molecular genetic basis; can be implemented by morphometric analysis; and, even with hematoxylin and eosin (H\&E) slides alone, has semiquantifiable features. ${ }^{[7]}$

The present study was aimed at reclassifying the previously diagnosed endometrial hyperplasia cases according to the WHO system in to the better reproducible EIN system.

\section{Materials \&Methods}

This is a retrospective study in which the endometrial curetting specimens done for patients with abnormal uterine bleeding, that were previously diagnosed as endometrial 
hyperplasia using the WHO94, from January 2014 to June 2016 were collected. Slides from all the patients were reviewed and recategorized according to the EIN system by the senior pathologist and the results were compared with the initial diagnosis.

Exclusion Criteria: Cases diagnosed as endometrial carcinoma, disorderly proliferative endometrium and complex hyperplasia with atypia in fractional curettage which were later diagnosed as endometrial carcinoma were excluded from the study.

\section{Results}

The total number of slides included for the study is 60 . The age group of patients was 32 to 63 years. Total number of fractional curettage specimen done on patients with abnormal uterine bleeding during this period was 708 and the incidence of benign hyperplasia among the patients with AUB is $5 \%$ and of EIN is $3 \%$ in our study.

Table 1shows the comparative diagnosis based on WHO and the EIN system with the number of patients. 38 out of the 46 cases $(82.6 \%)$ of simple hyperplasia were reclassified as Benign hyperplasia.8 out of the 46 cases (17.4\%) of simple hyperplasia, 1 case of complex hyperplasia without atypia and all the 11 cases of complex hyperplasia with atypia were reclassified as EIN.

Table 2 shows the no of patients affected according to the age group with the youngest patient being 32 year old and the oldest being 63 years and most of the patients $(66.7 \%)$ were in their fifth decade.

\section{Discussion}

Endometrial intraepithelial neoplasia (EIN) is a monoclonal premalignant endometrial glandular lesion that precedes the development of endometrioid-type endometrial adenocarcinoma. EIN arises through complex interactions involving the sequential accumulation of genetic damage in endometrial glands and the positive selective pressure of unopposed estrogen. Recent data have revealed a preclinical latent precursor lesion composed of mutated but morphologically nondescript glands that may persist for years in normal-appearing premenopausal cycling endometrium. This latent precursor shares many of the molecular features of EIN and endometrial adenocarcinoma, including frequent inactivation of both the tumor suppressor gene PTEN and the paired box-containing gene PAX2. Upon progression to EIN, the distinctive appearance of crowded and cytologically altered glands heralds a 45-fold increased risk of developing endometrial adenocarcinoma. To preserve the high predictability of EIN for concurrent/ subsequent adenocarcinoma, strict adherence to defined diagnostic criteria is essential. ${ }^{[8]}$

The diagnosis of EIN must meet 5 criteria in a single fragment, including architectural gland crowding, altered cytology, minimum size of $1 \mathrm{~mm}$, exclusion of carcinoma, and exclusion of mimics. The diagnosis of EIN can be summarized as a focus of clustered endometrial glands exceeding a gland to stroma ratio of $1: 1$, which have altered cytology from the background endometrium, and which comprise a sufficient volume of $1 \mathrm{~mm} \cdot{ }^{\left[{ }^{9}\right]}$

The new architectural criterion for EIN diagnosis, diminution of stromal volume to less than approximately half of the total sample volume, will also assist in discriminating between EH and EIN. Implementation of this proposal will bring diagnostic terminology into agreement with current concepts of premalignant endometrial disease and facilitate more uniform patient management.

Benign endometrial hyperplasia involves the entire endometrial compartment and, with protracted estrogen exposure, shows the progressive development of cysts, remodeled glands, vascular thrombi, and stromal microinfarcts. They are best construed as a sequence of changes whereby the appearance at any single time point is uniquely dependent on the preceding combination and the duration of hormonal exposures. In contrast, the

Table 2:

\begin{tabular}{|l|c|c|c|}
\hline WHO classification & No of cases & EIN classification & No of cases \\
\hline Simple hyperplasia & 46 & Benign hyperplasia & 38 \\
\hline Simple hyperplasia with atypia & 2 & Endometrial intraepithelial hyperplasia & 22 \\
\hline Complex hyperplasia & 1 & & \\
\hline Complex hyperplasia with atypia & 11 & & \\
\cline { 1 - 2 } Total no of cases : $\mathbf{6 0}$
\end{tabular}

Table 3:

\begin{tabular}{|c|c|}
\hline Age group & No of patients \\
\hline $31-40$ years & 13 \\
\hline $41-50$ years & 40 \\
\hline $51-60$ years & 6 \\
\hline $61-70$ years & 1 \\
\hline
\end{tabular}




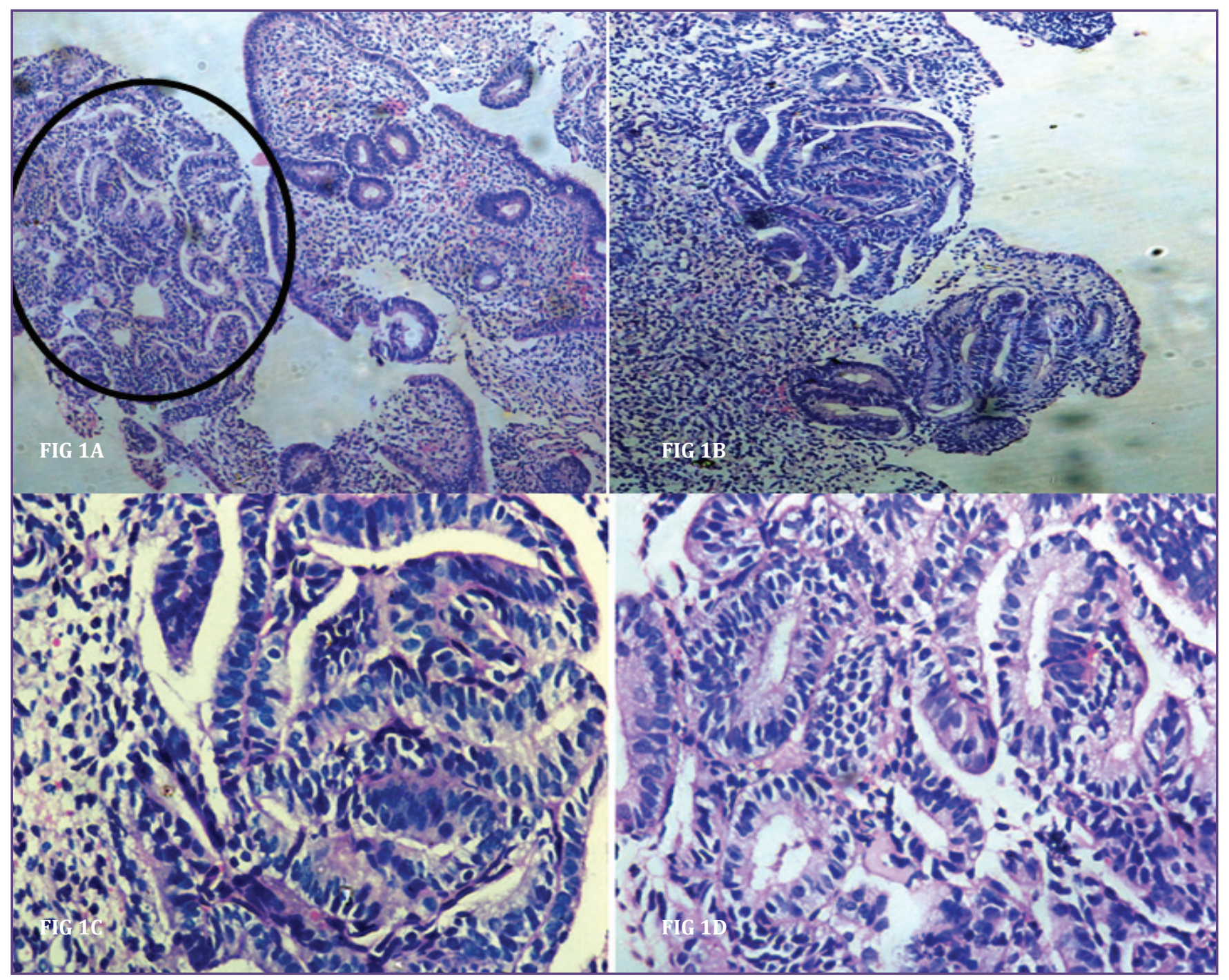

Fig. $1 \mathrm{~A}$ : Photomicrograph shows a focus of EIN with crowding of glands with cytological atypia (circled area )in contrast with the background benign glands( arrow),H\&E,X100; 1 B : Photomicrograph shows a focus of EIN with crowding of glands ,H\&E,X400; C \&D : Photomicrographs shows a focus of EIN with cytological atypia,H\&E,X400.

premalignant clone of an EIN lesion is characteristically offset from the background endometrium by its altered cytology and crowded architecture. The use of an internal standard for cytology assessment, combined with the distinctive topography of a clonal process, enables the diagnosis of EIN lesions with a long-term cancer risk 45 -fold greater than that of their benign endometrial hyperplasia counterparts. The resolution of hormonal and premalignant subsets of traditional "endometrial hyperplasias" is possible using redefined diagnostic criteria, enabling patient therapy to be appropriately matched with the underlying disease mechanisms. ${ }^{[10]}$

The EIN system, in contrast, has a molecular genetic basis; can be implemented by morphometric analysis; and even with hematoxylin and eosin (H\&E) slides alone, has semiquantifiable features. ${ }^{[11]}$

From the table 1 , it is very clear that categorization of endometrial hyperplasia in to two tier using the EIN system is much simpler and easily reproducible by the clinicians to stratify patients in to low risk and high risk groups for much easier follow up.

In a Cox regression analysis, EIN was the strongest prognostic index of future endometrial carcinoma. A longterm follow-up study of 477 women with hyperplasia done by Baak et al, suggest that the EIN classification scheme predicts the development of future malignancies more accurately than the WHO system. ${ }^{[11]}$ 
One of the major strengths of the EIN system is its correlation to outcome data. As discussed, a biopsy diagnosis of EIN imparts a 45-fold increased risk of progression to carcinoma after the first year. Hysterectomy following the diagnosis of EIN is appropriate because there is a high rate of concurrent, as well as future, endometrioid endometrial carcinoma in women with EIN. In circumstances in which the patient desires fertility or is not a surgical candidate, progestin therapy is an increasingly offered alternative. Progestin regimens are not standardized, and clinical outcomes are primarily available from anecdotal series rather than controlled randomized clinical trials. A common practice following progestin administration is a follow-up biopsy every 6 months following withdrawal until a minimum of 3 negative biopsies areobtained. ${ }^{[1]}$

\section{Conclusion:}

The results of this study show that the EIN classification scheme is superior to the WHO94 scheme in discriminating lesions with the highest risk for conversion to malignant disease. Furthermore, a large group of women who initially are diagnosed with "hyperplasia" but are classified later without EIN have a near-negligible risk of developing malignant disease.

Application of criteria for diagnosis of EIN successfully segregates patients into high and low cancer risk subgroups with better reproducibility than atypical hyperplasia diagnosis based on WHO system.

Acknowledgement: I thank Dr. Arulmalar Neeyam, tutor of our department for helping me in this study.

\section{References}

1. Owings RA; Quick CM, Endometrial Intraepithelial Neoplasia, Archives of Pathology \& Laboratory medicine, 2014, 138, 138:484-491

2. Hecht JL, Ince TA, Baak JPA, Baker HE, Ogden MW, Mutter GL. Prediction of endometrial carcinoma by subjective endometrial intraepithelial neoplasia diagnosis. Mod Pathol. 2008;18(3):324-330.

3. Baak JP, Mutter GL, Robboy S, et al. The molecular genetics and morphometry-based endometrial intraepithelial neoplasia classification system predicts disease progression in endometrial hyperplasia more accurately than the 1994 World Health Organization classification system. Cancer.2005;103(11):2304-2312.

4. Orbo A, Baak JPA, Kleivan I, et al. Computerized morphometrical analysis in endometrial hyperplasia for the prediction of cancer development: a longterm retrospective study from northern Norway. J ClinPathol.2006;53(9):697-703.

5. Baak JP, Orbo A, van Diest PJ, et al. Prospective multicenter evaluation of the morphometric D-score for prediction of the outcome of endometrial hyperplasias. Am J SurgPathol.2007, 25: 930-935.

6. Marie-Claude Renaud, Quebec QC, Tien Le, MD, Ottawa ON. Epidemiology and Investigations for suspected Endometrial Cancer, JointSoGC-GoC-SCC Clinical practice Guideline, 2013 291,

7. Silverberg SG, Kurman RJ, Nogales F, Mutter GL, KubikHuch RA, Tavassoli FA. Epithelial tumors and related lesions of endometrium. In: TavassoliFA, StrattonMR, editors. Tumors of the breast and female genital organs. Lyon: IARC Press, 2003: 221-232.

8. Zaino RJ, Kauderer J, Trimble CL, et al. Reproducibility of the diagnosis of atypical endometrial hyperplasia: a Gynecologic Oncology Group study. Cancer.2006;106(4):804-811.

9. Marotti JD, Glatz K, Parkash V, Hecht JL. International internet-based assessment of observer variability for diagnostically challenging endometrial biopsies. Arch Pathol Lab Med. 2011;135(4):464-470.

10. Allison KH, Reed SD, Voigt LF, Jordan CD, Newton KM, Garcia RL.Diagnosing endometrial hyperplasia: why is it so difficult to agree?Am J SurgPathol.2008; 32(5):691-698.

11. Mutter GL, Zaino RJ, Baak JP a, Bentley RC, Robboy SJ. Benign endometrial hyperplasia sequence and endometrial intraepithelial neoplasia.Int J GynecolPathol. 2007;26(2):103-114

*Corresponding author:

Rajalakshmi.V, Department of Pathology, ESIC Medical College \& PGIMSR, Chennai, Tamilnadu,India.

Email: raji_path@rediffmail.com

Date of Submission : 12.08.2016

Date of Acceptance : 14.04.2017

Financial or other Competing Interests: None.

Date of Publication : 31.08.2017 